\title{
The effect of high level multi-tone excitation on the acoustic properties of perforates and liner samples
}

\author{
Hans Bodén \\ Linné Flow Centre \\ MWL, Aeronautical and Vehicle Engineering, KTH, \\ S-100 44 Stockholm, Sweden
}

\begin{abstract}
This paper discusses the effect of high level multi-tone acoustic excitation on the acoustic properties of perforates and liner samples. It is based on a large experimental study of the nonlinear properties of these types of samples without mean grazing or bias flow. It is known from previous studies that high level acoustic excitation at one frequency will change the acoustic impedance of perforates at other frequencies, thereby changing the boundary condition seen by the acoustic waves. This effect could be used to change the impedance boundary conditions and for instance increase the absorption. It could obviously also pose a problem for the correct modelling of sound transmission through ducts lined with such impedance surfaces. First a quasi-stationary model for the acoustic properties of the perforate is discussed and the results are compared to experimental data. The effect of the combination of frequency components in the excitation signal is studied to find out if it matters if we are using tones which are harmonically related or not. The effect the phase of the frequency components is studied using both the model and experimental data. It is also discussed if a parameter controlling the impedance can be found for an arbitrary combination of tones with different frequencies.
\end{abstract}

\section{Introduction}

$\mathrm{T}$ here is a large number of papers on the effect of high level acoustic excitation on the acoustic properties of perforates and orifice plates ${ }^{1-17}$. Orifice plates and perforates appear in many technical applications where they are exposed to a combination of high acoustic excitation levels and either grazing or bias flow or a combination. Examples are automotive mufflers and aircraft engine liners. It is well known from the literature that perforates can become non-linear at fairly low acoustic excitation levels. The non-linear losses are associated with vortex shedding at the outlet side of the orifice or perforate openings. In the linear case the impedance is independent of the sound field but when the sound pressure level is high the perforate impedance will be dependent on the acoustic particle velocity in the holes. For pure tone excitation the impedance will be controlled by the acoustic particle velocity at that frequency. If the acoustic excitation is random or periodic with multiple harmonics the impedance at a certain frequency will depend on the particle velocity at other frequencies ${ }^{17}$. The present paper discusses the effect of high level multi-tone acoustic excitation on the acoustic properties of perforates and liner samples. It is based on a large experimental study of the nonlinear properties of these types of samples without mean grazing or bias flow. It is known from previous studies that high level acoustic excitation at one frequency will change the acoustic impedance of perforates at other frequencies, thereby changing the boundary condition seen by the acoustic waves. This effect could be used to change the impedance boundary conditions and for instance increase the absorption, as in the socalled zero mass-flow liner ${ }^{18}$. This effect can also pose a problem for the correct modelling of sound transmission through ducts lined with such impedance surfaces. The present paper will attempt to answer some questions which are important for the understanding these effects such as: Does the combination of frequencies matter? It could for instance make a difference if we are using tones which are harmonically related or not. Does the phase of the frequency components make a difference? This could be yet another factor to use for changing the impedance. Can a parameter controlling the impedance be found which controls the impedance for an arbitrary combination of tones with different frequencies? One possible choice could be the total particle velocity at the sample surface. 


\section{Perforate impedance model}

The model used here takes the starting point in the model presented by Cummings ${ }^{7}$. This model can excluding bias flow effects be written

$$
l(t) \frac{d V(t)}{d t}+\frac{1}{2}\left(\frac{V(t)}{C_{D}}\right) \cdot\left|\frac{V(t)}{C_{D}}\right|=\frac{\Delta p(t)}{\rho_{0}},
$$

where $l(t)$ is an effective orifice thickness including end corrections which can be time varying, $V(t)$ is the fluctuating acoustic velocity in the orifice, $C_{D}$ is a discharge coefficient to consider the vena contracta effect and $\Delta p(t)$ is the fluctuating pressure difference over the orifice. In the model according to Eq. (1) linear viscous losses are neglected. Such a term can be added either from theory ${ }^{16}$ or from experimental data giving

$$
l(t) \frac{d V(t)}{d t}+\frac{1}{2}\left(\frac{V(t)}{C_{D}}\right) \cdot\left|\frac{V(t)}{C_{D}}\right|+R_{L} V(t)=\frac{\Delta p(t)}{\rho_{0}} .
$$

In the article by Cummings ${ }^{7}$ an empirical expression for the time varying effective orifice length was presented

$$
l(t)=l_{0}+\frac{l_{W}+l_{0}}{1+\frac{\left(L_{V}(t) / d\right)^{1.585}}{3}},
$$

where $l_{0}$ is the end correction on one side of the orifice, $l_{W}$ is the orifice length, $L_{V}(t)$ is a time varying jet length caused by the high level acoustic excitation and $d$ is the orifice diameter. Cummings suggested that the jet length should be estimated from

$$
L_{V}(t)=\int_{0}^{\tau} V(t) d t
$$

where $\tau$ is the time from the beginning of the previous acoustic half cycle after $V(t)$ has changed sign. Here the result of using Eq. (4) has been compared to the assumption that $L_{V}$ does not vary with time but only with the level of acoustic excitation such that

$$
L_{V}=\int_{0}^{T / 2}|V(t)| d t
$$

It was found by comparison with experimental data that a modified version of Eq. (3), shown in Eq. (6), gave a better agreement with experimental results for some perforates. This has, together with jet length estimation using Eq. (5) been used for some of the simulation studies presented in section IIII.

$$
l(t)=l_{0}+\frac{l_{W}+l_{0}}{1+\frac{\left(L_{V}(t) / d\right)^{1.2}}{24}}
$$

\section{Experimental setup}

Most of the experimental data presented were obtained using an impedance tube with the perforate sample mounted at the end and an open termination behind the perforate sample. The sample was mounted using a holder causing a 
slight area reduction. The impedance change caused by the holder was measured separately and deducted from the perforate sample results. Three quarter inch condenser microphones were used with microphone separations $0.05 \mathrm{~m}$ and $0.3 \mathrm{~m}$. The distance between the sample and the nearest microphone was $0.15 \mathrm{~m}$. Some data was also used from a test setup according to Fig. 1 were a single orifice was mounted in a duct with two microphones on each side. The test object was an orifice plate with $3 \mathrm{~mm}$ thickness and $6 \mathrm{~mm}$ hole diameter. The orifice plate was mounted in a rigid tube with a diameter of $40 \mathrm{~mm}$. On the left hand side, a high quality loudspeaker was mounted as the excitation source. The microphone separation was in this case $0.18 \mathrm{~m}$. This test rig was used to study the combination of high acoustic excitation levels and bias flow ${ }^{20}$ but only no flow results will be presented here.

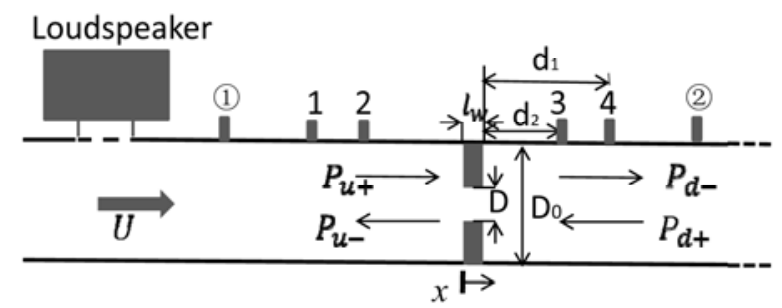

Figure 1. Test rig for measurement of orifice impedance.

Tonal excitation was used either with single tone excitation or with a combination of tones with different frequencies. Measurements in the impedance tube were made for four different circular hole perforate samples, as specified in Table 1. The perforate samples studied are described in Table 1 in terms of their hole diameter $(d)$, plate thickness $(t)$ and porosity $(\sigma)$. Results obtained for sample P4 with the smallest porosity will mainly be presented in this paper. Measurements were in the impedance tube also made for a couple of realistic aircraft engine liner samples.

Table 1. Specification of circular hole perforate samples tested in the impedance tube.

\begin{tabular}{|l|l|l|l|}
\hline Sample & $\mathrm{d}[\mathrm{mm}]$ & $\mathrm{t}[\mathrm{mm}]$ & $\sigma$ \\
\hline P1 & 3 & 1 & 0.28 \\
\hline P2 & 2 & 1.5 & 0.032 \\
\hline P3 & 2 & 2 & 0.086 \\
\hline P4 & 1 & 2 & 0.020 \\
\hline
\end{tabular}

\section{Results and discussion}

\section{A. Single tone excitation}

Figure 4 shows an example of a typical result obtained using single tone excitation. The real and imaginary parts of the normalised impedance have been plotted against the inverse Strouhal number based on the peak particle velocity in the holes and the hole diameter $(1 / S t=|\hat{V}| / \omega d)$. It can be seen that at an inverse Strouhal number close to one the acoustic properties start to become nonlinear and change with the level of excitation. 


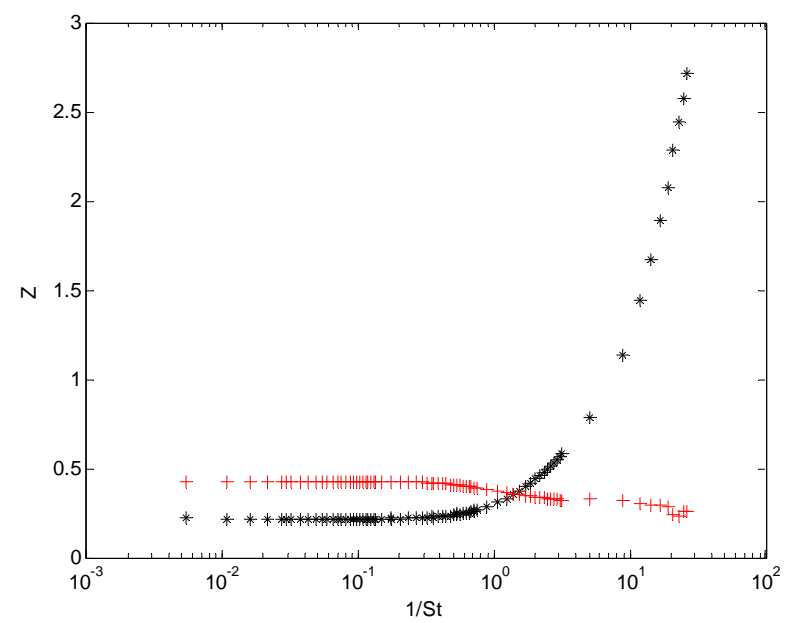

Figure 2. Normalized impedance for perforate sample P4 with $2 \%$ porosity, plotted against inverse Strouhal number: black stars - real part, red - plus imaginary part.

Equation (2) was solved in the time domain using the assumptions regarding the jet length according to Eq. (4) and Eq. (5) for different levels of single tone excitation. The pressure difference was assumed to be sinusoidal and the equation was solved to obtain $V(t)$. The impedance at the frequency of excitation was then obtained by taking the FFT of $\Delta p$ and $V$ and then dividing the two. These results were then compared to measured impedances. Figure 3 shows results at $200 \mathrm{~Hz}$ for the single orifice with $2.25 \%$ porosity.

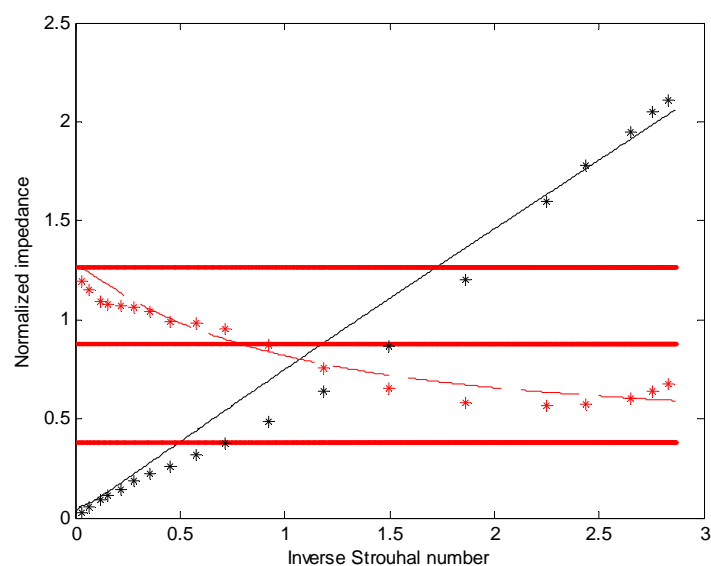

a)

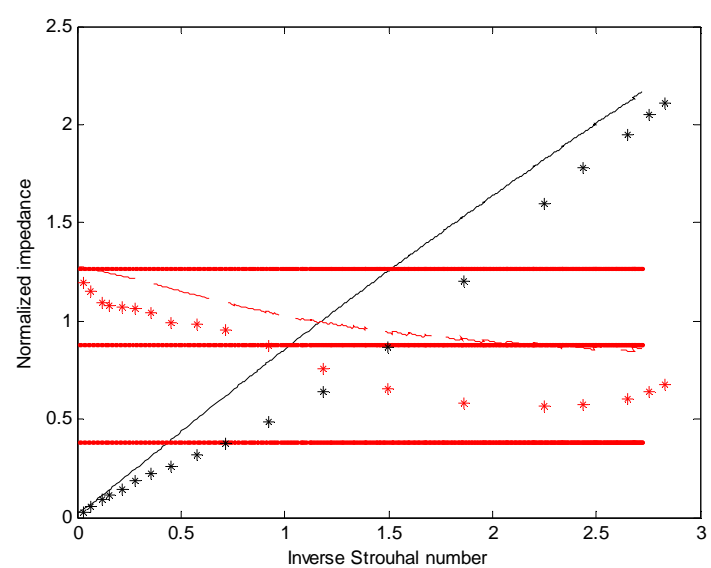

b)

Figure 3. Normalized impedance for single orifice with $2.25 \%$ porosity, plotted against inverse Strouhal number: black stars - measured real part, red stars - measured imaginary part, black solid line - simulated real part, red dashed line - simulated imaginary part, red thick lines - imaginary parts obtained assuming: $I=I_{0}, I=I_{0}+I_{W}$ and $I=2 I_{0}+I_{W}$. a) Jet length according to Eq. (5), b) jet length according to Eq. (4).

It can be seen that the best agreement is obtained using the jet length according to Eq. (5) while the assumption according to Eq. (4) gives an under prediction of the drop in effective orifice length caused by the high level acoustic excitation. Figure 4 show the corresponding results for perforate sample P4 with $2 \%$ porosity where now Eq. (6) has been used together with Eq. (5). 


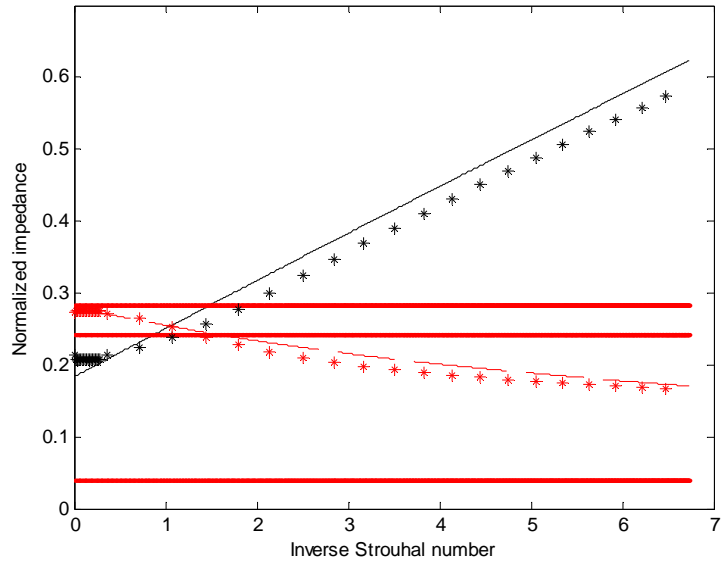

a)

Figure 4. Normalized impedance for perforate with $2 \%$ porosity, plotted against inverse Strouhal number: black stars - measured real part, red stars - measured imaginary part, black solid line - simulated real part, red dashed line - simulated imaginary part, red thick lines - imaginary parts obtained assuming: $I=I_{0}, I=I_{0}+I_{W}$ and $l=2 I_{0}+I_{W}$. a) Jet length according to Eq. (5), b) jet length according to Eq. (4).

It can be seen that for this case the assumption of Eq. (5) together with Eq. (6) gives a better agreement with experimental data. The model can give reasonable results when compared to experimental data and will therefore be used to complement experimental results in the subsequent sections. The time varying jet length estimation according to Eq. (4) gives small jumps in the results. This problem gets worse when the excitation is multi tone rather than single tone. For the perforate sample P4 with $2 \%$ porosity which will be studied the model with time varying effective length according to Eq. (5) and (6) will be used. The time variation of the effective length during one cycle for the highest level of excitation is shown in Fig, (5) and the variation of effective length with level of excitation obtained when the result using Eq. (5) is inserted in Eq. (3) is shown in Fig 6.

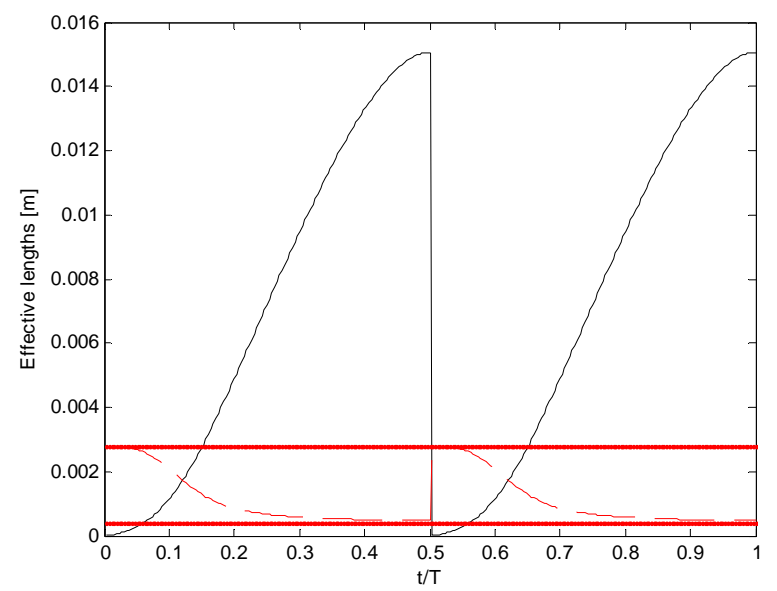

Figure 5. Time variation of jet length and effective orifice length according to Eq. (4) and Eq. (3) for a perforate with $2 \%$ porosity and for the highest level of acoustic excitation according to Fig. (4): black solid line - jet length, red dashed line - effective orifice length, red thick lines - imaginary parts obtained assuming: $I=I_{0}$ and $I=2 l_{0}+I_{W}$.

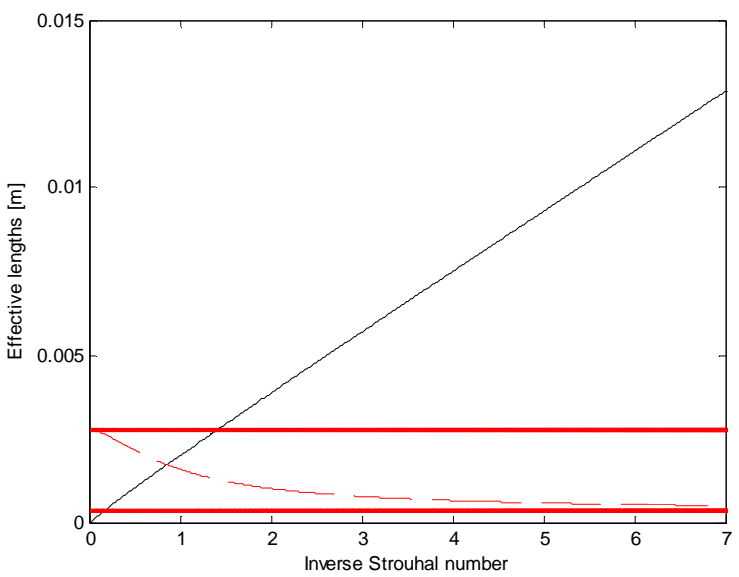

Figure 6. Variation of jet length and effective orifice length according to Eq. (5) and Eq. (3) for a perforate with $2 \%$ porosity for different levels of acoustic excitation: black solid line - jet length, red dashed line effective orifice length, red thick lines - imaginary parts obtained assuming: $l=l_{0}$ and $l=2 l_{0}+l_{W}$. 


\section{B. Multi tone excitation}

In this section experimental and simulation results for multi tone excitation are presented. Figure 7 shows the variation of measured impedance, for a $2 \%$ porosity perforate, at $330 \mathrm{~Hz}$ and $110 \mathrm{~Hz}$ when the excitation at the other frequency is varied. For comparison the impedance obtained using single tone excitation is also included. The resistance increases as the level of secondary tone excitation increases while the reactance decreases. For very high secondary tone excitation levels the impedance will go to minus the impedance seen from the sample back towards the impedance tube ${ }^{21}$, so the resistance will have a maximum and then drop down and become negative.
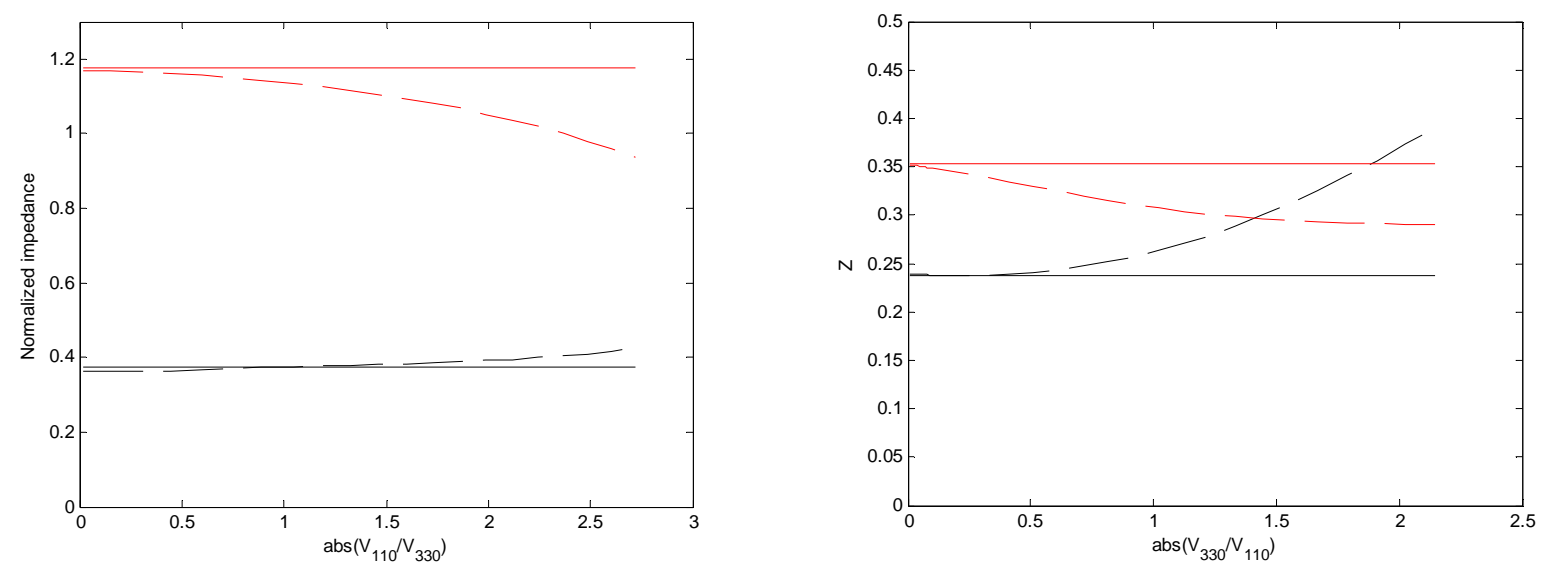

Figure 7. Measured normalized impedance for a perforate with $2 \%$ porosity, plotted against the ratio between velocities at $110 \mathrm{~Hz}$ and $330 \mathrm{~Hz}$ : a) impedance at $330 \mathrm{~Hz}$ : black solid line - real part excitation only at $330 \mathrm{~Hz}$, red solid line - imaginary part excitation only at $330 \mathrm{~Hz}$, black dashed line - real part excitation at $110 \mathrm{~Hz}$ and $330 \mathrm{~Hz}$, red dashed line - imaginary part excitation at $110 \mathrm{~Hz}$ and $330 \mathrm{~Hz}$, b) impedance at 110 Hz: black solid line - real part excitation only at $110 \mathrm{~Hz}$, red solid line - imaginary part excitation only at $110 \mathrm{~Hz}$, black dashed line - real part excitation at $330 \mathrm{~Hz}$ and $110 \mathrm{~Hz}$, red dashed line - imaginary part excitation at $330 \mathrm{~Hz}$ and $110 \mathrm{~Hz}$.

Figures 8 and 9 shows comparisons between experimental results and simulations using Eqs. (2), (5) and (6). In Fig. (8) the variation of impedance at $330 \mathrm{~Hz}$ when the level of excitation at $110 \mathrm{~Hz}$ is varied while the level of excitation at $330 \mathrm{~Hz}$ is kept constant is shown. Curves for different levels of excitation at $330 \mathrm{~Hz}$ are shown for the simulation results. Figure 9 shows the corresponding results at $110 \mathrm{~Hz}$ when the level of excitation at $330 \mathrm{~Hz}$ is varied. It can be seen that the simulation model gives reasonable agreement with the experimental results which means that it can be used to study trends caused by other types of variation in the excitation. 


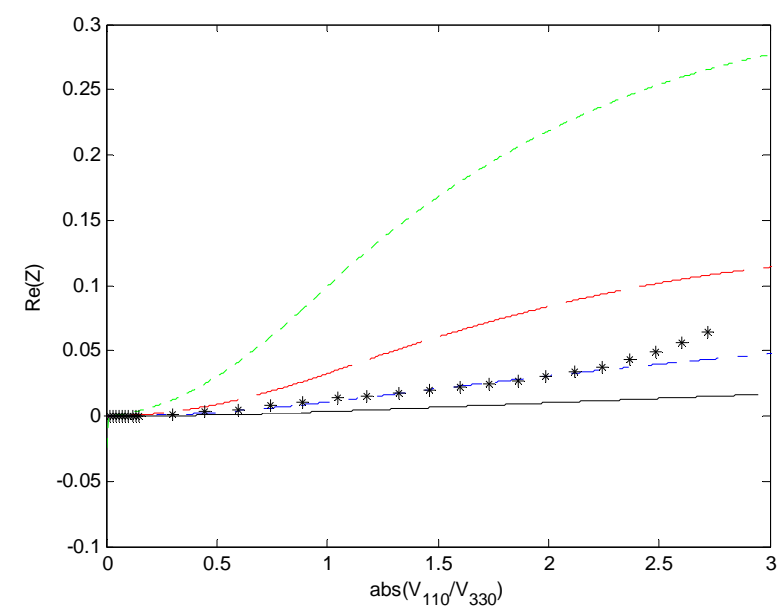

a)

Figure 8. Normalized impedance for perforate with $2 \%$ porosity at $330 \mathrm{~Hz}$, for varying level of excitation at $110 \mathrm{~Hz}$ while the level of excitation at $330 \mathrm{~Hz}$ is kept constant and with impedance for excitation at $330 \mathrm{~Hz}$ only removed, plotted against ratio between velocity at $110 \mathrm{~Hz}$ and $330 \mathrm{~Hz}$. Excitation levels at $330 \mathrm{~Hz}$ : black solid line - simulated $0.06 \mathrm{~m} / \mathrm{s}$, blue dashed-dotted line - simulated $0.2 \mathrm{~m} / \mathrm{s}$, red dashed line - simulated 0.6 $\mathrm{m} / \mathrm{s}$, green dotted line - simulated $2 \mathrm{~m} / \mathrm{s}$, stars - measured $0.6 \mathrm{~m} / \mathrm{s}$. a) Real part, b) imaginary part.

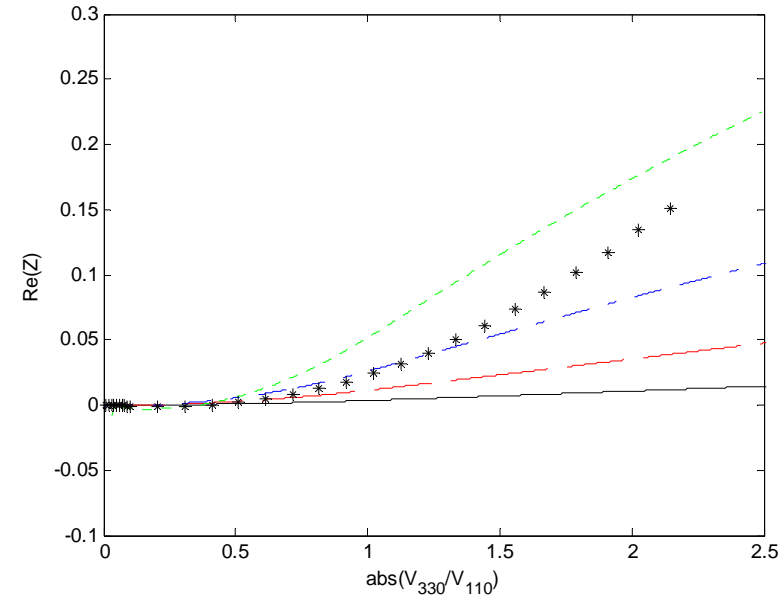

a)

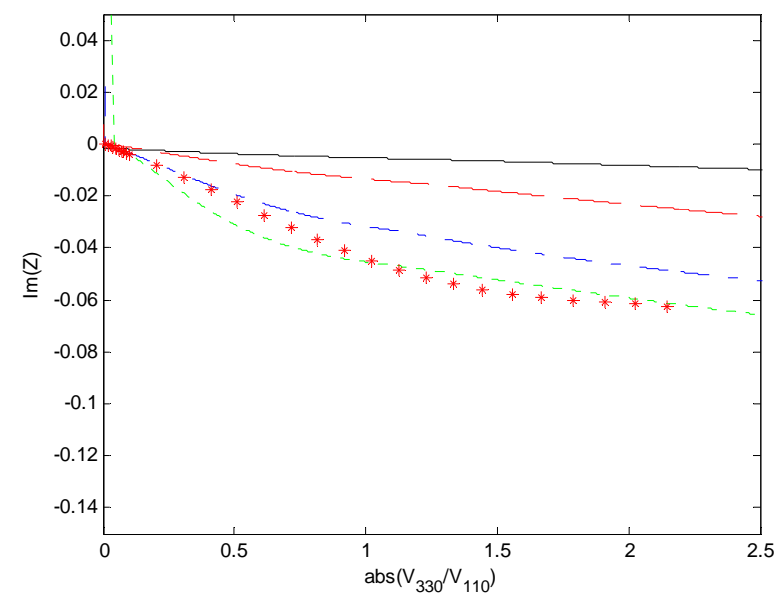

b)

Figure 9. Normalized impedance for perforate with $2 \%$ porosity at $110 \mathrm{~Hz}$, for varying level of excitation at $330 \mathrm{~Hz}$ while the level of excitation at $110 \mathrm{~Hz}$ is kept constant and with impedance for excitation at $110 \mathrm{~Hz}$ only removed, plotted against ratio between velocity at $330 \mathrm{~Hz}$ and $110 \mathrm{~Hz}$. Excitation levels at $110 \mathrm{~Hz}$ : black solid line - simulated $0.07 \mathrm{~m} / \mathrm{s}$, blue dashed-dotted line - simulated $0.2 \mathrm{~m} / \mathrm{s}$, red dashed line - simulated 0.6 $\mathrm{m} / \mathrm{s}$, green dotted line - measured $2.1 \mathrm{~m} / \mathrm{s}$. a) Real part, b) imaginary part.

\section{Multi tone excitation - effect of combination of frequencies}

Figure 10 shows normalized impedance results obtained using multi-tone excitation on sample P4 with $2 \%$ porosity, plotted against total peak particle velocity in the holes. Measurements were made with single tone excitation, two-tone excitation, three-tone excitation, four tone excitation and five-tone excitation. The impedance results are also compared to two different semi-empirical models described $i^{15,17}$. It can be seen that there is a large 
scatter in the results showing that the total, summed, peak particle velocity is not the only parameter controlling the results.
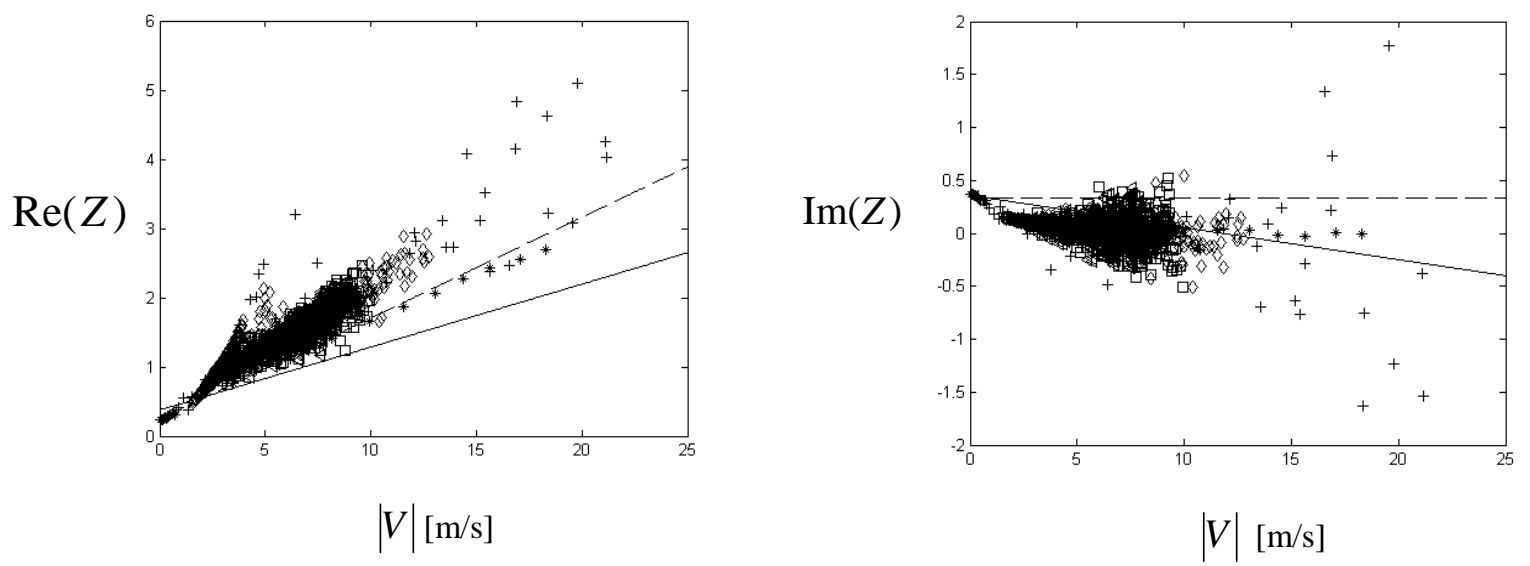

Figure 10. Normalized perforate impedance for sample $P 4$ at $110 \mathrm{~Hz}$ as a function of peak particle velocity in the holes; left resistance, right reactance, measured using different number of tones; stars - single tone, + two tones, diamonds - three tones, squares - four tones, < - five tones, straight line- theory according to ${ }^{15}$, dashed line - theory described in ${ }^{17}$.

Measurements have also been made on an aircraft engine liner sample using multi-tone techniques. Figure 11 shows normalised impedance results at $990 \mathrm{~Hz}$ obtained with different combinations of excitation frequencies. It can be seen that the effect of varying the level at $330 \mathrm{~Hz}$ has the largest effect consistent with the result that nonlinear energy transfer mainly occurs to higher order odd harmonics. The effect should therefore be largest at three times the excitation frequency. In the curve where the level of excitation at $330 \mathrm{~Hz}$ is kept constant while the level of excitation at $990 \mathrm{~Hz}$ is varied we can see that with low level of excitation at $990 \mathrm{~Hz}$ we get a negative resistance because the nonlinear energy transfer from $330 \mathrm{~Hz}$ dominates the result. As the level of excitation at $990 \mathrm{~Hz}$ is increased we approach the resistance obtained for single tone excitation at $990 \mathrm{~Hz}$, without changing the total particle velocity much, since it is dominated by the excitation at $330 \mathrm{~Hz}$. These results show that it is difficult to find a single parameter controlling the obtained impedance results.

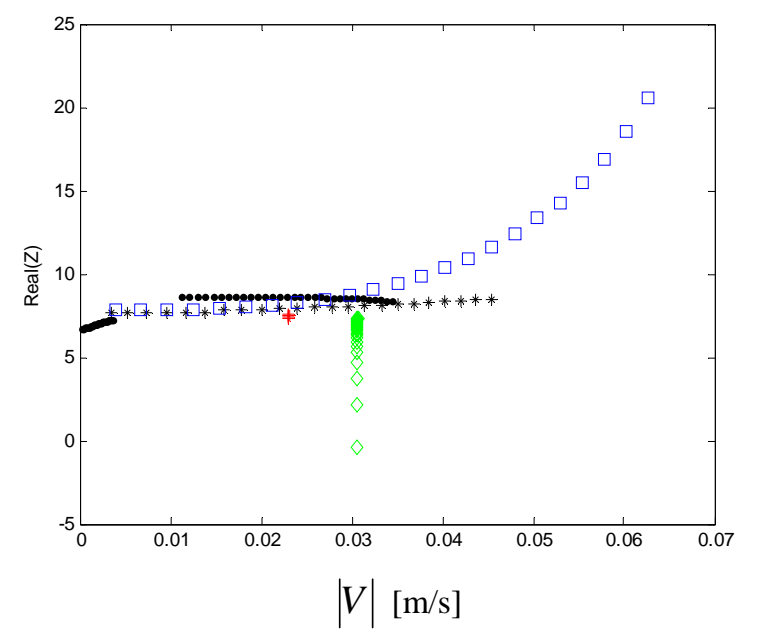

Figure 11. Real part of normalized perforate impedance for liner sample at $990 \mathrm{~Hz}$ as a function of peak particle velocity incident on the sample, measured using different combinations of tones; black dots - single tone excitation at $990 \mathrm{~Hz}$, black stars - excitation at $990 \mathrm{~Hz}$ constant and excitation at $110 \mathrm{~Hz}$ varied, blue boxes - exci6tation at $990 \mathrm{~Hz}$ constant and excitation at $330 \mathrm{~Hz}$ varied, red plus - excitation at $110 \mathrm{~Hz}$ constant and excitation at $990 \mathrm{~Hz}$ varied, green diamonds - excitation at $330 \mathrm{~Hz}$ constant and excitation at $990 \mathrm{~Hz}$ varied. 
Figure 12 presents the results of another experiment where it was studied if it makes a difference for the results obtained using two-tone excitation if the frequencies used are exact harmonics or not. It can be seen that the largest variation is obtained when excitation at $110 \mathrm{~Hz}$ interacts with the result at $330 \mathrm{~Hz}$. This again shows that the combination of frequencies does make a difference and that excitation at harmonics have a larger effect compared to excitation at other frequencies.

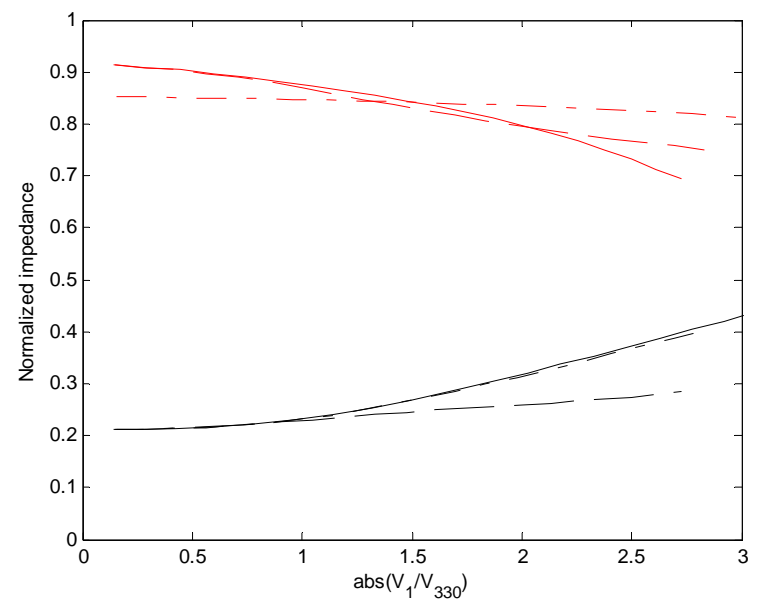

a)

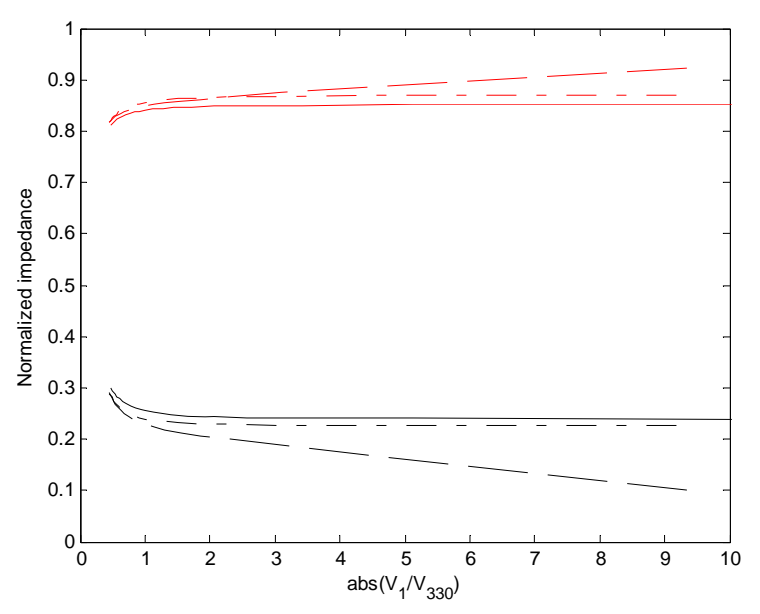

b)

Figure 12. Normalized impedance for perforate with $2 \%$ porosity at $330 \mathrm{~Hz}$ plotted against velocity ratio: Black - real part, red - imaginary part, solid line $-V_{1}=V_{100}$, dashed line $-V_{1}=V_{110}$, dashed-dotted line - $V_{1}$ $=V_{120}$. a) Excitation at $330 \mathrm{~Hz}$ varied and excitation at the other frequencies kept constant, b) excitation at $100 \mathrm{~Hz}, 110 \mathrm{~Hz}$ and $120 \mathrm{~Hz}$ varied and excitation at $330 \mathrm{~Hz}$ kept constant.

\section{Multi tone excitation - effect of phase}

In order to test if changing the phase of the higher harmonics has an influence on the result a standard impedance tube tests have been made with excitation at $110 \mathrm{~Hz}$ and $330 \mathrm{~Hz}$. The level of excitation was varied either by changing the excitation level at $110 \mathrm{~Hz}$ or $330 \mathrm{~Hz}$. At each excitation level the phase at $330 \mathrm{~Hz}$ was shifted 180 degrees. Figures 13 and 14 shows comparisons of experimental results and simulation results. In Fig. 13 the normalized impedance at $330 \mathrm{~Hz}$ obtained when the level of excitation at $110 \mathrm{~Hz}$ was varied is show for cases where the phase at $330 \mathrm{~Hz}$ is varied. In the measurements the phase was shifted by 180 degrees while a few additional results are shown in the simulation results. Figure 14 shows the corresponding results at $110 \mathrm{~Hz}$. When comparing the experimental and simulated results it should be noticed that the reference case shown as solid lines do not have exactly the same phase in the experiments and simulations. What should be compared is the magnitude of the difference caused by a phase shift. It can be seen that this variation is predicted well by the simulation. The phase relation between the tones is of importance at least at higher ratios between the velocities. 

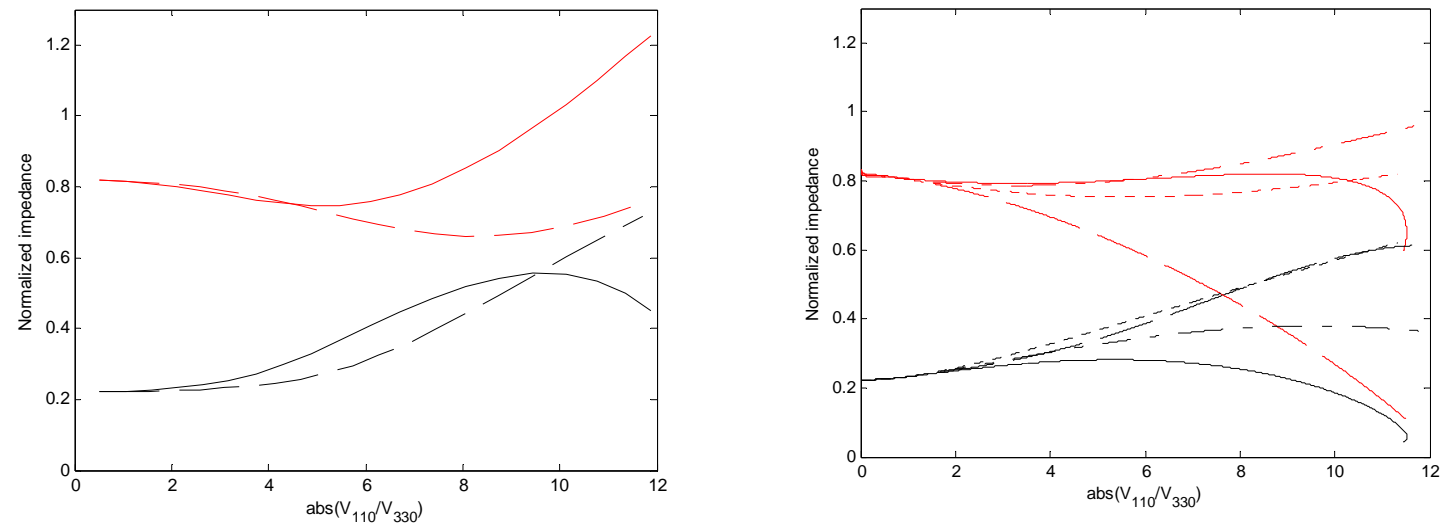

Figure 13. Real and imaginary parts of normalised impedance at $330 \mathrm{~Hz}$ for a perforate sample with $2 \%$ porosity plotted against velocity ratio for excitation at $110 \mathrm{~Hz}$ varied and excitation at $330 \mathrm{~Hz}$ kept constant: black - real part, red - imaginary part, solid line - phase at $330 \mathrm{~Hz}$ not shifted , dashed - phase at $330 \mathrm{~Hz}$ shifted 180 degrees, dashed-dotted - phase at $330 \mathrm{~Hz}$ shifted 60 degrees, dotted - phase at $330 \mathrm{~Hz}$ shifted 90 degrees. a) Measured, b) simulation.
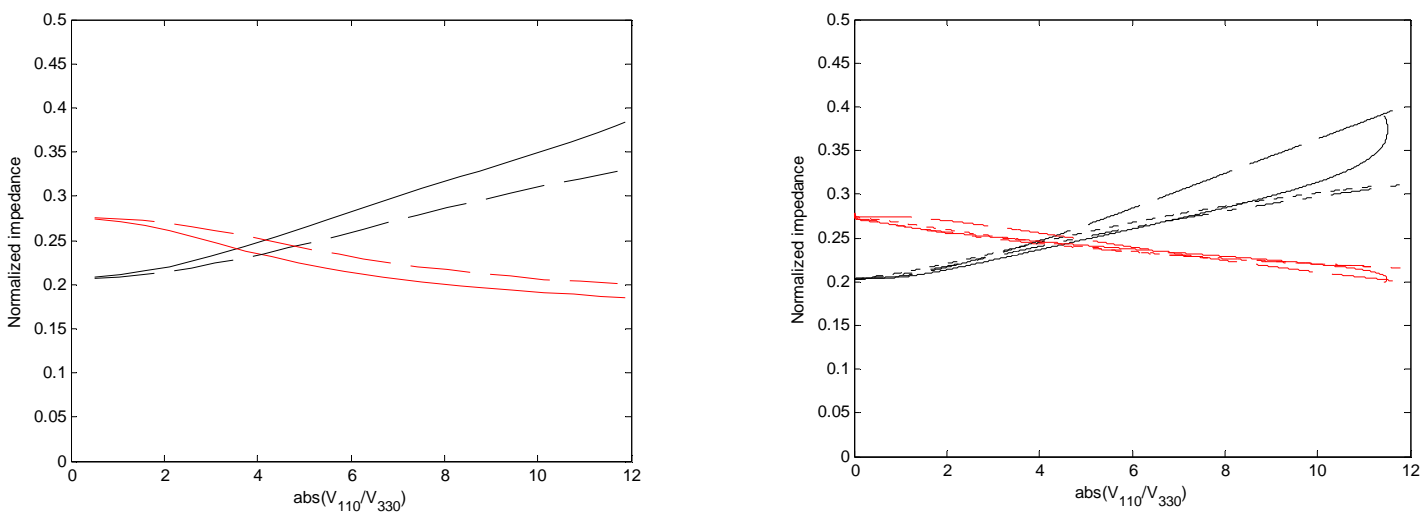

Figure 14. Real and imaginary parts of normalised impedance at $110 \mathrm{~Hz}$ for a perforate sample with $2 \%$ porosity plotted against velocity ratio for excitation at $110 \mathrm{~Hz}$ varied and excitation at $330 \mathrm{~Hz}$ kept constant: black - real part, red - imaginary part, solid line - phase at $330 \mathrm{~Hz}$ not shifted , dashed - phase at $330 \mathrm{~Hz}$ shifted 180 degrees, dashed-dotted - phase at $330 \mathrm{~Hz}$ shifted 60 degrees, dotted - phase at $330 \mathrm{~Hz}$ shifted 90 degrees. a) Measured, b) simulation

\section{Conclusions}

The result of high level acoustic multi-tone excitation on the acoustic properties of perforates and liner samples has been investigated experimentally and using a model. It was shown that a modified version of the Cummings ${ }^{7}$ model gives sufficiently good results when compared to experimental data to use the model for parameter variation studies. It was concluded that the combination of frequencies is of importance for multi tone excitation and that harmonically related tones have a stronger influence on the nonlinear interaction results compared to other combinations of frequencies. The phase of the tones used also makes a difference for the result. It was concluded that no single parameter which controls the obtained impedance results for an arbitrary combination of tones could be found. 


\section{References}

${ }^{1}$ Sivian I.J., Acoustic impedance of small orifices. Journal of the Acoustical Society of America 7, 1935 ,pp. 94-101.

${ }^{2}$ Ingård, U. and Labate, S., "Acoustic circulation effects and the nonlinear impedance of orifices", Journal of the Acoustical Society of America, Vol. 22, 1950, pp. 211-219.

${ }^{3}$ Ingård, U. and Ising, H., "Acoustic nonlinearity of an orifice", Journal of the Acoustical Society of America, Vol. 42, 1967, pp. 6-17.

${ }^{4}$ Melling, T.H., "The acoustic impedance of perforates at medium and high sound pressure levels”, Journal of Sound and Vibration, Vol. 29, No. 1, 1973, pp. 1-65.

${ }^{5}$ Zinn B.T.. "A theoretical study of nonlinear damping by Helmholtz resonators.” Journal of Sound and Vibration 13, 1970, pp. 347-356.

${ }^{6}$ Cummings A., “Acoustic nonlinearities and power losses at orifices.” AIAA Journal 22(6), 786-792 (1984).

${ }^{7}$ A. Cummings 1986. Journal of the Acoustical Society of America 79, 942-951. Transient and multiple frequency sound transmission through perforated plates at high amplitude.

${ }^{8}$ Salikuddin, M. "Acoustic behaviour of orifice plates and perforated plates with reference to low-frequency sound absorption.” Journal of Sound and Vibration 139, 361-381 (1990).

${ }^{9}$ Salikuddin M. and Brown W.H., "Non-linear effects in finite amplitude wave propagation and perforated plate terminations.” Journal of Sound and Vibration 139, 383-405 (1990).

${ }^{10}$ Panton R.L. and Goldman L., "Correlation of nonlinear orifice impedance.” Journal of the Acoustical Society of America 60, 1390-1396 (1976).

${ }^{11}$ Ingård, U., "Nonlinear distortion of sound transmitted through an orifice", Journal of the Acoustical Society of America, Vol. 48, 1970, pp. 32-33.

${ }^{12}$ Tam, C.K.W. and Kurbatski, K.A., "Micro-fluid dynamics and acoustics of resonant liners”, AIAA Paper, AIAA 99-1850, 1999.

${ }^{13}$ Tam, C.K.W., Kurbatski, K.A., Ahuja K.K.and Gaeta Jr., R.J. “A Numerical and Experimental Investigation of the Dissipation Mechanisms of Resonant Acoustic Liners”, Journal of Sound and Vibration, Vol. 245, No. 3, 2001, pp. 545-557.

${ }^{14}$ Tam, C.K.W., Ju, H., Jones, M.G, Watson, W.R. and Parrott, T.L. “A Computational and Experimental Study of Slit Resonators”, Journal of Sound and Vibration, Vol. 284, No. 3-5, 2005, pp. 947-984.

${ }^{15}$ Elnady, T. and Bodén, H., “On semi-empirical liner impedance modeling with grazing flow”, AIAA Paper, AIAA 2003-3304, May 2003.

${ }^{16}$ Elnady, T., "Modelling and characterization of perforates in lined ducts and mufflers (Paper III)", PhD Thesis, Department of Aeronautical and Vehicle Engineering, KTH, Stockholm, Sweden, 2004.

${ }^{17}$ Bodén, H., Ying, G. and Tözün, H.B., "Experimental investigation of nonlinear acoustic properties for perforates”, AIAA Paper 2006- 2404.

${ }^{18}$ Lahiri C., Pardowitz B., Bake F., Röhle I. and Enghardt L., "Excitation of a Zero Mass Flow Liner for Acoustic Damping”, AIAA Journal, vol. 49, issue 3, pp. 513-519.

${ }^{20}$ Bodén, H. and Zhou , L. "Acoustic properties of an in-duct orifice subjected to bias flow and high level acoustic excitation”, Proceedings of FIV 2012.

${ }^{21}$ Bodén, H. "One-sided multi-port techniques for characterisation of in-duct samples with nonlinear acoustic properties”, Journal of Sound and Vibration, Vol. 331, 2012, pp. 3050-3067. 